05. 1;06.5;10

\title{
Влияние механических напряжений в пластине из алюминиевого сплава Д16 на генерацию акустических колебаний лазерным излучением
}

\author{
(С) А.Л. Глазов, К.Л. Муратиков \\ Физико-технический институт им. А.Ф. Иоффе РАН, Санкт-Петербург, Россия \\ E-mail: glazov.holo@mail.ioffe.ru
}

Поступило в Редакцию 24 мая 2019г.

В окончательной редакции 4 июня 2019г.

Принято к публикации 4 июня 2019г.

\begin{abstract}
Проанализирован процесс лазерной генерации ультразвука в металлах с внутренними механическими напряжениями. Исследованы особенности поведения фотоакустического сигнала вблизи отверстия в пластине из сплава Д16 при воздействии механических напряжений. Выявлено несоответствие экспериментальных результатов предсказаниям термодинамической модели зависимости коэффициента теплового расширения от механических напряжений. Для корректного описания особенностей поведения сигнала в реальных металлах предложено учесть влияние электронной подсистемы на лазерную генерацию акустических колебаний.
\end{abstract}

Ключевые слова: термоупругость, фотоакустическая микроскопия, остаточные напряжения, задача Кирша, давление электронного газа.

DOI: 10.21883/PJTF.2019.17.48226.17892

Лазерные ультразвуковые и фотоакустические (ФА) методы с успехом используются для получения информации о механических, термоупругих и теплофизических свойствах объемных материалов, твердотельных структур и тонких пленок. Отдельный интерес вызывает применение методов лазерной ФА-микроскопии для диагностики внутренних напряжений в различных материалах. В настоящее время анализ данных лазерных ФА-экспериментов проводится на основе классической теории термоупругости, которая предполагает общий подход к материалам различной природы. В частности, такая теория не делает различий между диэлектриками и металлами [1], в то время как металлы в отличие от диэлектриков наряду с атомами решетки имеют электронную подсистему. Это различие может существенно влиять на термоупругий эффект при быстрых термодинамических процессах. Такая ситуация имеет место в металлах при воздействии коротких лазерных импульсов, например, из-за так называемого эффекта „электронного взрыва“ [2]. Кроме того, экспериментально показано, что при лазерном воздействии наблюдаются различия в возбуждении акустического отклика в металлах и диэлектриках. В частности, показано, что при воздействии наносекундного лазерного импульса термоупругий отклик в металлических образцах длится намного дольше, чем в диэлектриках, и не может быть описан в рамках классический модели термоупругости [3-5]. В [6] приведен обзор и детально теоретически рассмотрен ФА-эффект в полупроводниках. В работе [7] было предложено использовать двухтемпературную модель оптического возбуждения акустических волн в проводниках. Однако данные работы не позволяют оценить влияние напряжений на ФА-эффект в проводниках.
Для анализа особенностей образования лазерных ФА-сигналов в металлах в настоящей работе нами экспериментально и теоретически исследовано поведение таких сигналов вблизи отверстий. Достоинство подобного подхода состоит в наличии точных аналитических решений для распределения напряжений вблизи отверстия в пластине [8]. Кроме того, метод сверления отверстий для диагностики напряжений в металлах в сочетании с интерферометрическим или тензометрическим методами хорошо отработан $[9,10]$, что позволяет сравнить результаты, полученные этими методами и ФА-методом.

Схема экспериментальной установки описана, например, в [11]. В рамках настоящей работы экспериментальные исследования были выполнены на образце из алюминиевого сплава Д16 с поверхностью $5.9 \times 5.6 \mathrm{~mm}$ и толщиной $2.7 \mathrm{~mm} \mathrm{c} \mathrm{малым} \mathrm{отверстием} \mathrm{в} \mathrm{центре}$ диаметром $0.27 \pm 0.02 \mathrm{~mm}$. Во время измерений имелась возможность прикладывать к образцу одноосное сжимающее напряжение. Изменение внешней нагрузки производилось в пределах области упругих деформаций.

Генерация акустических волн в образце осуществлялась периодически меняющимся градиентом температуры вследствие поглощения модулированного во времени лазерного излучения. Диаметр луча на поверхности образца $20 \mu \mathrm{m}$. Длина тепловой волны в образце на рабочей частоте модуляции $141 \mathrm{kHz}$ составляла $15 \mu \mathrm{m}$. Регистрация ФА-сигнала от образца осуществлялась пьезоэлектрическим датчиком, подсоединенным к его тыльной стороне. При сканировании лазерным лучом поверхности образца условия генерации ФА-сигнала оставались неизменными, за исключением области шириной около $30 \mu \mathrm{m}$ вблизи края отверстия, где имело место отражение тепловых волн. 


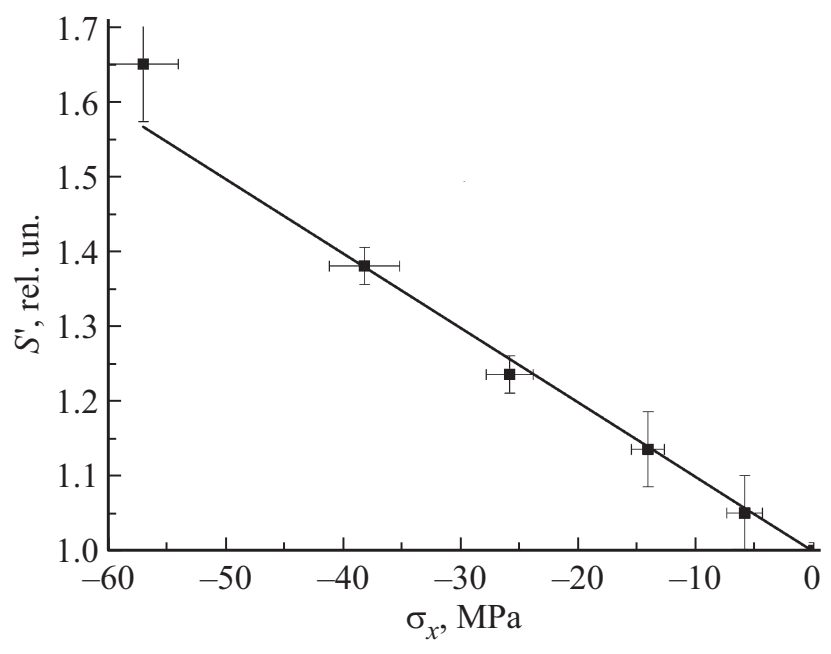

Рис. 1. Зависимость усредненного сигнала вдали от отверстия от приложенного давления.

Зависимость нормированного усредненного ФА-сигнала от внешнего сжимающего напряжения $\sigma_{x}$ вдали от отверстия показана на рис. 1. Сплошная линия на рис. 1 соответствует линейной зависимости сигнала $S^{\prime}=1+b \sigma_{x}$ при $b=-(0.0100 \pm 0.0004) \mathrm{MPa}^{-1}$.

Рассмотрим теперь характер изменения ФА-изображений области вблизи отверстия при приложении одноосного напряжения. Амплитудные ФА-изображения для ненагруженного и нагруженного образца приведены на рис. 2. На рис. 2, $a$ распределение ФА-сигнала в целом однородное, за исключением узкой области около края отверстия, что соответствует отсутствию в пластине сильных внутренних напряжений в исходном состоянии. На изображении (рис. $2, b$ ), полученном при сжимающем напряжении $57 \pm 3 \mathrm{MPa}$, видны диаметрально расположенные светлые и темные области, соответствующие известному распределению внутренних напряжений в пластине с отверстием радиуса $a$ при одноосном нагружении. Такое распределение в полярных координатах $r$ и $\vartheta$ описывается в соответствии с решением задачи Кирша [12]

$$
\sigma(r, \vartheta)=\sigma_{x}\left(1-2 a^{2} \cos (2 \vartheta) / r^{2}\right) .
$$

Рассмотрим поведение ФА-сигнала в зависимости от полярного угла. Для радиуса $0.15 \mathrm{~mm}$ эта зависимость представлена на рис. 3. Сплошной линией показан результат аппроксимации данных функцией $p_{1}+p_{2} \cos \left(2\left(\vartheta-p_{3}\right)\right)$ с подгоночными параметрами $p_{i}$, т.е. в линейном приближении зависимости $S(\sigma)$. Эта функция хорошо описывает периодичность изменения сигнала. При этом имеют место некоторые отклонения амплитуды ФА-сигнала от выбранной функции, связанные с незначительным отклонением от линейности, которые в настоящей работе не рассматриваются.

По аналогии с [11] для определения зависимости ФА-сигнала от напряжений проведем аппроксимацию
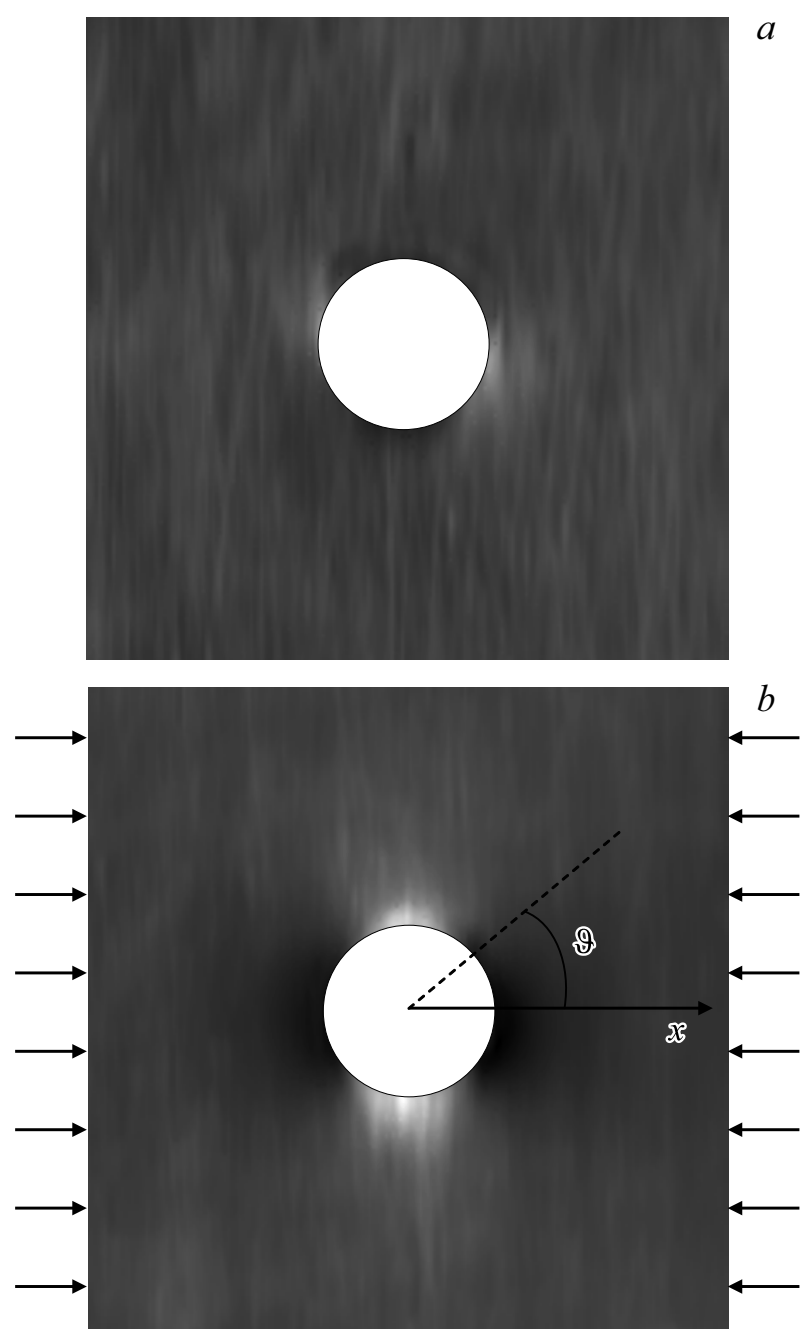

Рис. 2. ФА-изображения части образца из сплава Д16 вокруг отверстия. $a$ - для образца без внешнего давления, $b-$ под одноосной нагрузкой $57 \mathrm{MPa}$. Размер изображений $0.95 \times 0.95 \mathrm{~mm}$. Частота колебаний $141 \mathrm{kHz}$.

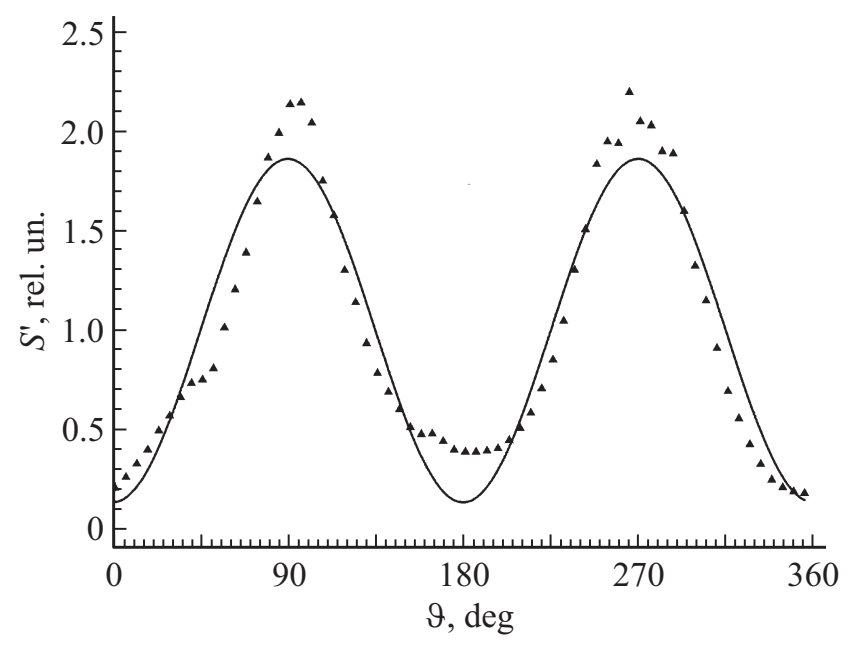

Рис. 3. Изменение ФА-сигнала вдоль окружности с радиусом $0.15 \mathrm{~mm}$. Кривая - результат подгонки функцией $p_{1}+p_{2} \cos \left(2\left(\vartheta-p_{3}\right)\right)$. 
экспериментальных данных модельной функцией вида

$$
S^{\prime}(r, \vartheta)=S(r, \vartheta) /\langle S\rangle=1-A \cos \left(2\left(\vartheta-\vartheta_{0}\right)\right) / r^{2}
$$

для области $150<r<500 \mu \mathrm{m}$. Здесь $\langle S\rangle=S\left(\sigma_{x}\right)-$ усредненная амплитуда сигнала вдали от отверстия, а угол $\vartheta_{0}$ учитывает несовпадение направлений сканирования и действия напряжения. В peзультате были получены следующие значения параметров: $A=16960 \pm 30 \mu \mathrm{m}^{2}, \vartheta_{0}=-0.0086 \pm 0.0001 \mathrm{rad}$. Отсюда, полагая радиус $a=140 \pm 10 \mu \mathrm{m}$, получаем $S(r, \vartheta)=S_{0}\left(1-(1.5 \pm 0.4) \cdot 10^{-2} \sigma(r, \vartheta)\right)$, где $\sigma$ выражается в МРа. Знание коэффициента перед $\sigma$ позволяет определять значение напряжения в данной области по величине ФА-сигнала.

При интерпретации данного результата следует обратить внимание на то, что значение полученного коэффициента $b$ для сплава Д16 имеет отрицательное значение. Однако в соответствии с известным термодинамическим соотношением, описывающим зависимость коэффициента теплового расширения от напряжений, он должен быть равен $b=(-\partial E / \partial T) /\left(E^{2} \alpha\right)$, где $\alpha-$ коэффициент теплового расширения, $E-$ модуль Юнга [13]. Поскольку для большинства материалов $\partial E / \partial T<0$, коэффициент $b$ должен быть положительным. Например, для сплава Д16 $E=71000 \mathrm{MPa}$, $\partial E / \partial T \approx-38 \mathrm{MPa} \cdot \mathrm{K}^{-1}, \alpha=23 \cdot 10^{-6} \mathrm{~K}^{-1}$, и соответственно $b \approx 3 \cdot 10^{-4} \mathrm{MPa}^{-1}$. Приведенная термодинамическая оценка предполагает неизменность внутренней структуры материала, достаточно однородное распределение по объему и медленное во времени тепловое и механическое воздействие.

В реальных металлах при наличии дефектов и неупорядоченных областей при внешних воздействиях может происходить существенная обратимая перестройка их электрон-решеточной структуры [14]. В этом случае концентрация свободных электронов в металле не остается неизменной, что приводит к изменению давления электронного газа $\delta p_{e}$. С точки зрения постановки задачи термоупругости необходимо в уравнение движения к источнику колебаний $(3 \lambda+2 \mu) \alpha \nabla T$ добавить еще одну силу $\nabla \delta P_{e}=\frac{2}{3} E_{\mathrm{F}} \nabla \delta n_{e}$, где $\lambda$ и $\mu-$ коэффициенты Ламе, $p_{e}$ - давление вырожденного электронного газа, $n_{e}-$ концентрация электронов, $E_{\mathrm{F}}-$ энергия Ферми. В условиях лазерных ФА-экспериментов осуществляется динамическое локальное тепловое воздействие на металл, которое может приводить к упомянутым процессам. Указанные процессы способны влиять на величину эффективного коэффициента теплового расширения металла, поэтому значение коэффициента $b$ и его знак при лазерных ФА-экспериментах могут не совпадать со значениями, определяемыми при статических термодинамических условиях.

Динамику концентрации электронов можно описать с помощью уравнения баланса частиц, где источник пред- ставлен в общем виде, используя закон Аррениуса [15]:

$$
\begin{aligned}
\frac{\partial \delta n_{e}(\mathbf{r}, t)}{\partial t} & +\frac{\delta n_{e}(\mathbf{r}, t)}{\tau}=A\left[\exp \left(-U / k\left(T_{0}+\delta T(\mathbf{r}, t)\right)\right)\right. \\
& \left.-\exp \left(-U / k T_{0}\right)\right]
\end{aligned}
$$

где $\tau$ - время релаксации всей системы, $U-$ энергия активации электрона, $k-$ постоянная Больцмана, $T_{0}$ - средняя температура нагретой области, $\delta T-$ переменная составляющая температуры. При наличии механических напряжений $U=U_{0}+V \sigma$, где $V$ - так называемый активационный объем.

Будем считать, что в условиях ФА-эксперимента $\delta T \ll T_{0}, \quad \delta T=\delta T(\mathbf{r}) \exp (i \omega t) . \quad$ Тогда правая часть уравнения (3) равна $A U \exp \left(-U / k T_{0}+i \omega t\right) \delta T(\mathbf{r}) / k T_{0}^{2}$. В этом случае переменная составляющая градиента концентрации зависит от $\sigma$ как

$$
\begin{aligned}
\nabla \delta n_{e}(\mathbf{r}, t)= & \frac{\tau A}{1+i \omega \tau} \exp \left(-\left(U_{0}+V \sigma+i \omega t\right) / k T_{0}\right) \\
& \times\left(U_{0}+V \sigma\right) / k T_{0}^{2} \nabla \delta T(\mathbf{r}) .
\end{aligned}
$$

Тогда коэффициент теплового расширения при малых напряжениях, таких, что $V \sigma \ll k T_{0}$, можно представить в виде

$$
\alpha_{e f f} \cong \alpha-\frac{1}{E^{2}} \frac{\partial E}{\partial T} \sigma-\frac{A V \tau\left(U_{0}-k T_{0}\right)}{k T_{0}^{2}(1+i \omega \tau)} \sigma \exp \left(-U_{0} / k T_{0}\right) .
$$

Здесь $\alpha_{e f f}-$ эффективный коэффициент теплового расширения в отсутствие напряжений. Выражение (4) имеет смысл при $U_{0}>k T_{0}$, так как в обратном случае электроны дефекта делокализованы уже в исходном состоянии. Тогда второе и третье слагаемые в выражении (5) имеют разные знаки, т. е. зависимость коэффициента теплового расширения от напряжения может отличаться от предсказываемого термодинамической моделью. Таким образом, предложенная модель лазерной генерации звука позволяет качественно объяснить экспериментальные данные для реальных металлов.

Сравнение экспериментальных данных с термодинамической моделью термоупругой генерации акустических колебаний показало, что обычный подход с учетом только теплового расширения решетки не позволяет правильно описать зависимость ФА-сигнала от внутренних напряжений. Для корректного описания лазерной генерации ультразвуковых колебаний в реальных металлах предложено учитывать электронные процессы, зависящие как от температуры, так и от деформаций. Для этого в уравнение движения добавлены силы, зависящие от градиента давления электронного газа, которые могут приводить к увеличению ФА-сигнала при сжимающих напряжениях в соответствии с данными эксперимента.

\section{Конфликт интересов}

Авторы заявляют, что у них нет конфликта интересов. 


\section{Список литературы}

[1] Nowacki W. Thermoelasticity. Oxford: Pergamon Press, 1986. $578 \mathrm{p}$.

[2] Tzou D.Y., Chen J.K., Beraun J.E. // J. Thermal Stresses. 2005. V. 28. N 6-7. P. 563-594. doi.org/10.1080/01495730590929359

[3] Sudenkov Yu.V., Zimin B.A. // Int. J. Heat Mass Transfer. 2015. V. 85. P. 781-786. doi.org/10.1016/j.ijheatmasstransfer.2015.01.119

[4] Зимин Б.А., Судьенков Ю.В. // Письма в ЖТФ. 2012. Т. 38. B. 24. C. $26-32$.

[5] Sudenkov Yu.V., Zimin B.A., Sventitskaya V.E. // AIP Conf. Proc. 2018. V. 1959. P. 070035 (1-8). doi.org/10.1063/1.5034710

[6] Гусев В.Э., Карабутов А.А. Лазерная оптоакустика. М.: Наука, 1991. $304 \mathrm{c}$

[7] Индейщев Д.А., Осипова Е.В. // ДАН. 2017. Т. 473. № 2. C. 1-5. doi.org/10.7868/S0869565217080084.

[8] Биргер И.А. Остаточные напряжения. М.: МАШГИЗ, 1963. $232 \mathrm{c}$.

[9] Apal'kov A.A., Odintsev I.N., Plotnikov A.S. // Inorgan. Mater. 2017. V. 53. N 15. P. 1496-1501. doi.org/10.1134/S0020168517150031

[10] Vitek K. // Appl. Mech. Mater. 2015. V. 732. P. 32-41. doi.org/10.4028/www.scientific.net/AMM.732.32

[11] Глазов А.Л., Морозов Н.Ф., Муратиков К.Л. // Физ. мезомеханика. 2019. Т. 22. № 2. С. 49-58.

[12] Glazov A.L., Morozov N.F., Muratikov K.L. // Doklady Physics. 2019. V. 64. N 1. P. 20-23.

[13] Bert C.W., Fu C. // J. Press. Vessel Technol. 1992. V. 114. P. 189-192.

[14] Eisenbach A., Havdala T., Delahaye J., Grenet T., Amir A., Frydman A. // Phys. Rev. Lett. 2016. V. 117. N 11. P. 116601 (1-5). doi.org/10.1103/ PhysRevLett.117.139901

[15] Rinn B., Maass P., Bouchaud J.-P. // Phys. Rev. B. 2001. V. 64. N 10. P. 104417. doi.org/10.1103/PhysRevB.64.104417 